\title{
Effect of E-cigarette Use and Social Network on Smoking Behavior Change: An agent-based model of E-cigarette and Cigarette Interaction
}

\author{
Yang Qin ${ }^{1}$, Rojiemiahd Edjoc ${ }^{2}$, and Nathaniel D Osgood ${ }^{1}$ \\ 1 University of Saskatchewan, Canada \\ first. last@usask.ca \\ 2 Statistics Canada, Ottawa, Canada \\ rojiemiahd.edjoc@canada.ca
}

\begin{abstract}
Despite a general reduction in smoking in many areas of the developed world, it remains one of the biggest public health threats. As an alternative to tobacco, the use of electronic cigarettes (ECig) has been increased dramatically over the last decade. ECig use is hypothesized to impact smoking behavior through several pathways, not only as a means of quitting cigarettes and lowering risk of relapse, but also as both an alternative nicotine delivery device to cigarettes, as a visible use of nicotine that can lead to imitative behavior in the form of smoking, and as a gateway nicotine delivery technology that can build high levels of nicotine tolerance and pave the way for initiation of smoking. Evidence regarding the effect of ECig use on smoking behavior change remains inconclusive. To address these challenges, we built an agent-based model (ABM) of smoking and ECig use to examine the effects of ECig use on smoking behavior change. The impact of social network (SN) on the initiation of smoking and ECig use were also explored. Findings from the simulation suggest that the use of ECig generates substantially lower prevalence of current smoker (PCS), which demonstrates the potential for reducing smoking and lowering the risk of relapse. The effects of proximity-based influences within SN increases the prevalence of current ECig user (PCEU). The model also suggests the importance of improved understanding of drivers in cessation and relapse in ECig use, in light of findings that such aspects of behavior change may notably influence smoking behavior change and burden.
\end{abstract}

Keywords: E-cigarette · Smoking · Agent-based modeling · Distancebased network

\section{Introduction}

Smoking and secondhand smoke harm nearly every organ of the body and contribute to many preventable diseases, including lung cancer, coronary heart disease, chronic obstructive pulmonary disease, and other cardiovascular diseases [6]17. Nicotine products come in various forms, e.g., cigarettes, nicotine gum, 
patch, and ECig [7]. ECigs, vaporizing a liquid mixture which is used as a substitute for tobacco leaves and stored inside cartridges [13 [16], were introduced to the market in 2003, promoted and marketed by major tobacco companies in the last decade [139. The use of ECig as a cigarette alternative has increased dramatically. The PCEU among US adults increased from $0.3 \%$ in 2010 to $6.8 \%$ in 2013 [8]. Within recent years, there has been a particularly dramatic and alarming rise in the use of ECig amongst youth.

The health behaviors associated with smoking have been studied in detail. The majority of smokers attempt to quit smoking, but fewer than $5 \%$ of them remain quit for more than three months [5]. Effective tools for smoking cessation (Sc) may help current smoker (CS) quit, and forestall an individual at risk of smoking, e.g., former smoker (FS), struggling with avoiding relapse. ECigs also allow never smoker (NS) seeking to experiment with nicotine as an alternative to cigarettes. The rise of ECig use is associated with a perception that ECig is safer than cigarettes and a useful Sc device. However, there remains little solid scientific evidence confirming the effectiveness and safeness of ECig as a SC tool 8120. By surveying 2028 US smokers in 2012 and 2014 and two-years of followup, Zhuang et al. [20] concluded that long-term ECig users had a higher rate of SC of $42.4 \%$ than short-term Ecig users and non-users (14.2\% and $15.6 \%$, respectively). Zhu et al. [19] concluded that ECig users have a higher rate of SC, and are more likely to remain quit than non-ECig users. Cherng et al. 8] proposed an ABM to exmaine the effect of ECig on the smoking prevalence of US adults, and concluded that the simulated effects of ECig on Sc largely changed smoking behavior. The ABM simulated the influences of smoking behavior on ECig use initiation and cessation, and how ECig reversely affected SC and smoking initiation SI.

While promising, previous studies have predominantly relied upon self reported surveys, cohort studies and clinical trials. Such larger studies are expensive, are associated with high delay until they show effect, and can be difficult to plan and execute given the wide variety of patterns of behavior possible (e.g., initiation of exclusive smoking following ECig use, initiation of exclusive ECig use following tobacco, dual use, start of ECig use following quitting tobacco, etc.). Clinical trials often regulate or exclude factors that play a key role in shaping outcomes in society, such as switching of nicotine delivery modality, varying rates of compliance, and peer influence effects.

In this paper, extending the preliminary model structure introduced by Cherng et al. 8], we build an ABM of smoking and ECig use with modalities of initiation, cessation, and relapse to examine the effects of ECig use on individual-level smoking behavior change and population-level smoking patterns according to the aggregation of individual outcomes. Our model incorporates strong SN effects involving both selection of networks and influence over networks, age, sex and history-dependent effects regarding the rate of initiation, cessation, and relapse for both smoking and ECig use, and individual decision-making effects based on characteristics of social contacts. In particular, we use the model to inves- 
tigate whether the ECig is an effective Sc device and the impact of ECigs on non-smokers with regards to SI.

\section{Methods}

Model Overview ABM can simulate complex social dynamics and behaviors with considerably high resolution, and generate population-level results by aggregating individual outcomes in different scenarios [8]. Equally notable, ABM is widely applied to probe the impacts of counter-factual interventions, as well as to help prioritize data collection in a complex milieu of complex interactions of behaviors and product types. In this study, a high level of heterogeneity characterizing both exogenous and endogenous components, specific traits at individual level and modularity also strongly motivated the use of ABM.

Our model was built in AnyLogic (version 8.3.3), and used four interacting statecharts for each agent, featuring smoking states, ECig use states, birth, and mortality. The parameters, transition rates and statecharts in the Person class serve as influences from within an agent on smoking and ECig use behavior. The model further incorporates a distance-based network to simulate social contacts between agents.

The model simulates a population of 100,000 agents with age distribution based on population pyramid of Canada [1. The model time unit is $1 \mathrm{yr}$, and the length of the time horizon is 70yrs. The initial states may misestimate the prevalence of each smoking and ECig use state, so a period of burn time (52 years) is used for the model to achieve equilibrium. Over the continuous time of the simulation, agents either maintain their current state of smoking and ECig use or transit to other status based the (hazard) rates discussed in the next section.

Model Formulation Smoking statechart describes three smoking states: never smoker (NS), CS and FS. An individual can switch its presence in each of the three states of statechart according to specified transition rates, namely the rate of SI, the rate of SC, and the rate of smoking relapse (SR).

ECig use statechart separates the states of ECig use as never ECig user (NEU), CEU and former ECig user (FEU). The transition of ECig use initiation (ECigUI) is fired with a hazard rate, transferring an agent from NEU to CEU. Other transitions are message triggered transitions, which will be activated only under scenarios when we consider: A CS who never used ECig may possibly initiate ECig use after quitting smoking, transiting from CS $\triangle$ NEU to FS $\triangle$ CEU by chance; An FS who is CEU may possibly quitting ECig after relapse to smoking, transferring from FS $\wedge$ CEU to CS $\wedge$ FEU by chance; And a CS who is FEU may possibly relapse to CEU after quitting smoking, transiting from $\mathrm{CS} \wedge$ FEU to FS $\wedge$ CEU by chance. For the two statecharts, agents can occupy a specific, concrete state of one statechart at any one time, while being in any state of the other statechart.

Rate of SI, SC and SR denoted as $r_{\mathrm{si}}, r_{\mathrm{sc}}$ and $r_{\mathrm{sr}}$, respectively, are each the product of its corresponding hazard rate $\left(\alpha_{\mathrm{si}}, \alpha_{\mathrm{sc}}\right.$ and $\alpha_{\mathrm{sr}}$ for the calculation of 
$r_{\mathrm{si}}, r_{\mathrm{sc}}$ and $r_{\mathrm{sr}}$, respectively), a multiplier $\left(m_{\mathrm{si}}, m_{\mathrm{sc}}\right.$ and $m_{\mathrm{sr}}$ for the calculation of $r_{\mathrm{si}}, r_{\mathrm{sc}}$ and $r_{\mathrm{sr}}$, respectively) and a coefficient $\left(e_{\mathrm{si}}, e_{\mathrm{sc}}\right.$ and $e_{\mathrm{sr}}$ for the calculation of $r_{\mathrm{si}}, r_{\mathrm{sc}}$ and $r_{\mathrm{sr}}$, respectively).

The hazard rates reflect the magnitude of the effect of age, gender and smoking history on $r_{\mathrm{si}}, r_{\mathrm{sc}}$ and $r_{\mathrm{sr}}$. We transformed the annual probabilities of SI and $\mathrm{SC}\left(p_{\mathrm{si}}\right.$ and $p_{\mathrm{sc}}$, respectively) of male and female of 1970 birth cohort, reported by Holford et al. [10, into their corresponding $\alpha_{\mathrm{si}}$ and $\alpha_{\mathrm{sc}}$ as table functions in AnyLogic by using $p=1-e^{-\alpha}$. The model assumed that $\alpha_{\text {sr }}$ declines with growing time since quit; thus, individuals who only recently quit have far higher relapse risk than an agent who has remained as FS for a prolonged period. The value of multipliers is driven by the state of ECig use. Wills et al. [18] suggested that NS who tried ECig is three times more likely to start smoking. Leventhal et al. 11] reported that ECig users were four times likely to uptake cigarettes. McRobbie et al. [12] suggested that the rate for SC was significantly higher in the presence of ECig use (RR 2.3; 95\%CI: 1.05 - 4.96). Based on the linkages between ECig use and $r_{\text {si }}$ and $r_{\text {sc }}$ mentioned above. As ECig use can help relieve the symptoms of nicotine withdrawal to some degree and might provide an additional avenue towards continued socialization with companions who remain tobacco users, CEUs are less likely to relapse in smoking, compared to non-ECig users [14]. Therefore the model assumes $m_{\mathrm{si}}$ is 4.0 for agents who are CS $\wedge$ CEU [11, or is 2.87 for agents who are FEU [18], $m_{\mathrm{sc}}$ is 2.3 for agents who are CS $\wedge$ CEU [12], $m_{\mathrm{sr}}$ is 0.5 for agents who are CS $\wedge$ CEU [14]. If each rate is only the product of its hazard rate and a multiplier, the rate may misestimate the projection of smoking. Therefore, the coefficients $e_{\mathrm{si}}, e_{\mathrm{sc}}$ and $e_{\mathrm{sr}}$ were calibrated to match simulation outcomes against historical data.

For the rate of ECiguI, the model adapted the time-based sigmoid function and divisors introduced by Cherng et al. [8], to characterize the increasing use of ECig after its introduction into the market and the influence of smoking status on ECigUI. Additionally, the rate of ECigUI is strongly related to the agent's smoking status and demographic factors [15], suggesting that ECig is popular in smokers and young people; thus, we assumed an hazard of ECigUI of male agents using a table function, which has a $\mathrm{x}$-axis of age of the agents and $\mathrm{y}$-axis of the hazard rate and follows same pattern as for the hazard rate of SI for male. If an individual is female, the hazard of ECigUI of this agent is given by the corresponding point on the table function divided by the variable divECigfemale with a value of 1.5. The overall rate of ECiguI is the product of the hazard of ECiguI given by the time-based sigmoid function [8] and a coefficient $\left(e_{\mathrm{ECig}}\right)$, which was calibrated by matching model generated incidence of ECig use against corresponding historical data.

The model assumes that the transition of ECig use cessation (ECiguc) and ECig use relapse (ECigUR) are affected only by the smoking behavior, that is, the model assumed that individuals who are CEUs or FEUs would remain so unless changes occurred in their smoking behavior. Specifically, in the absence of identified evidence with respect to the fraction of individuals whose state of ECig use will be affected by smoking behavior, the model posited that $85 \%$ of 
CEUS $\wedge$ FSs will quit ECig if they relapse to smoking, since their nicotine cravings were satisfied by smoking, and $80 \%$ of agents who are FEU $\wedge$ CS will transit to CEU if they quit smoking. As ECigs may be used as cessation tools, the model further assumed that $50 \%$ of smoking quitters would uptake ECig immediately after quitting smoking. Therefore, message dichotomous branching transitions were built for ECigUI and ECigUC under these assumptions in addition to the rate of ECiguI discussed above.

Age-specific birth and mortality rates drawn from Statistics Canada of 2016 [43] are used in the model. The total fertility rate of Canada in 2016 is 1.54 per woman. To maintain population replacement (with a total fertility rate of 2.1) for successive years of the model running, we thus multiplied a coefficient (with a value of 1.357 ) by the fertility rate of each age group.

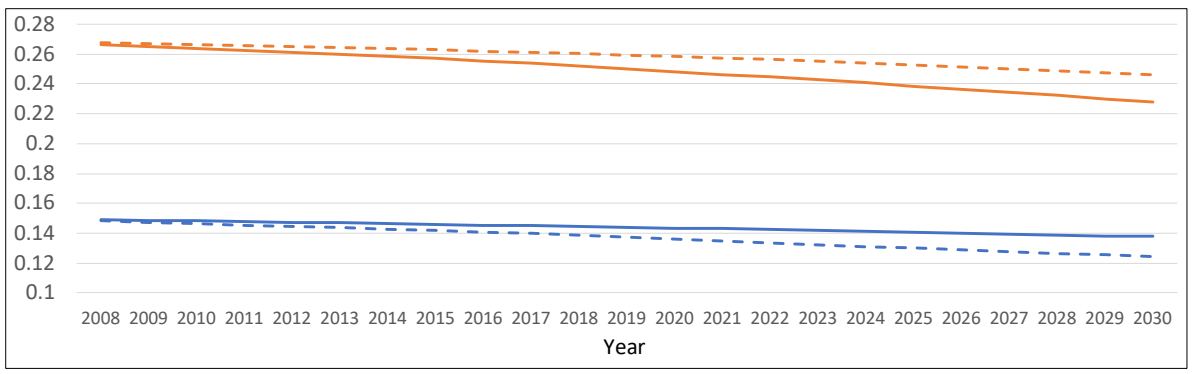

Fig. 1: PCS and PFS of Scn1 and Scn2. Orange and blue solid line represent the PFS and PCS in Scn1, respectively. Orange and blue dashed line represents the PFS and PCS in Scn2, respectively.

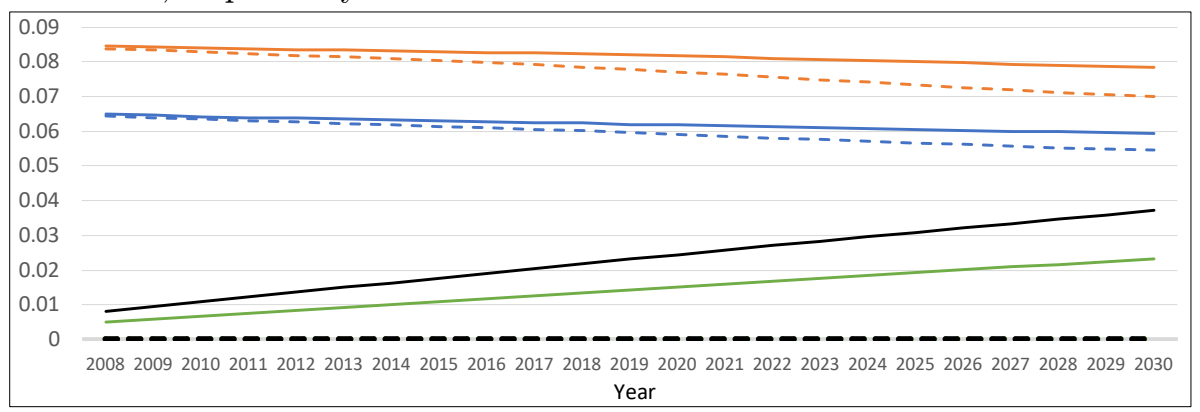

Fig. 2: PFCS, PMCS, PFCEU and PMCEU of Scn1 and Scn2. Blue, orange, green, and black solid line represent the PFCS and PMCS of Scn1, PFCEU and PMCEU of Scn2, respectively. Blue, orange, green, and black dashed line represent the PFCS and PMCS of Scn2, PFCEU and PMCEU of Scn1.

Smoking is well recognized as both an individual habit and a social phenomenon [5]. The baseline model was extended with a distance based network to simulate the effect of social connection and peer pressure on the SI and ECigUI. 
To build a localized SN for each agent, connecting with its nearby agents, the model assumed that an agent establishes the network with the agents in proximity $(50 \mathrm{~m})$. The $\mathrm{SN}$ was implemented as a dynamic network driven by agent mobility in continuous space with width and height both equal to $250,000 \mathrm{~m}$. Specifically, the agent moves to a new location within the space, and disconnects from the current network then re-establish a network based on agents layout by using a cyclic timeout event with an interval of 2 yrs. As dynamic network, the fraction of CS and CEUs among its connected agents are modified with the change of the SN, therefore, influence the effect of SN on SI and ECigUI.

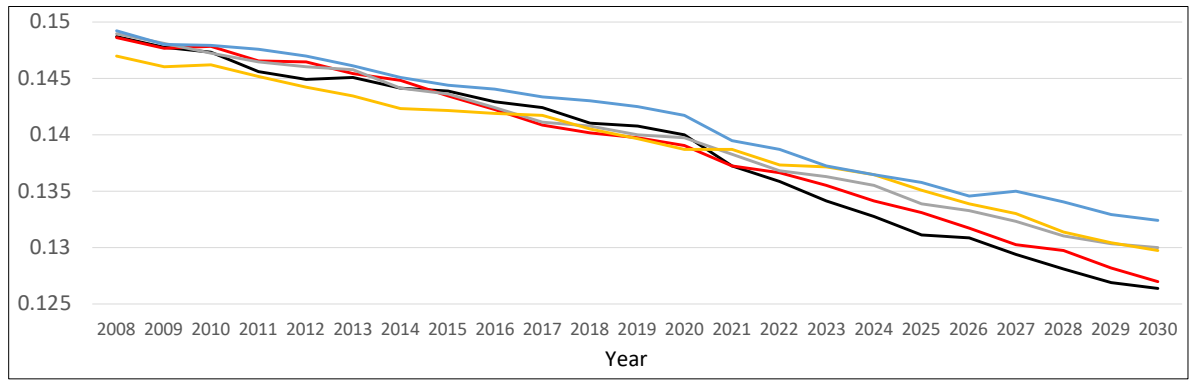

Fig. 3: SA of rate of ECigUC on PCS. Black, red, grey, yellow, and blue line represent a successively larger rate of ECiguC of $0.2,0.4,0.6,0.8$, and 1.0, respectively.

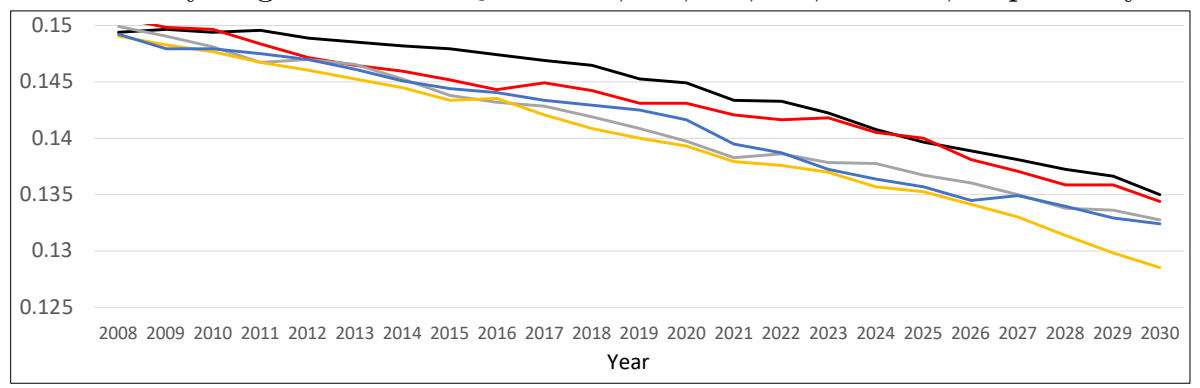

Fig. 4: SA of rate of ECigUR on PCS. Black, red, grey, yellow, blue line represent a successively larger rate of ECigUR of $0.2,0.4,0.6,0.8,1.0$, respectively.

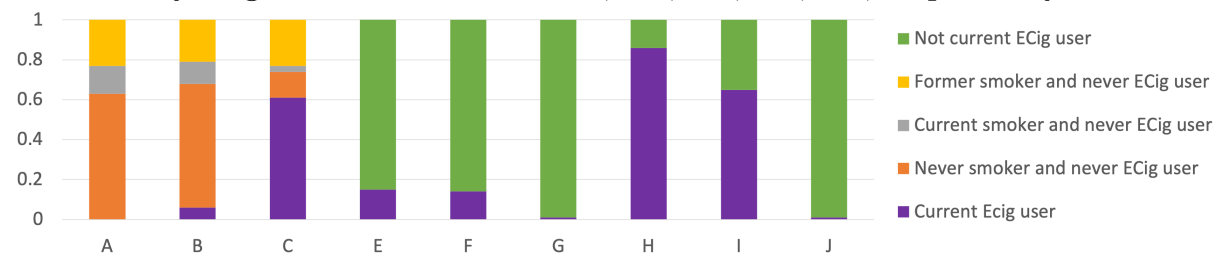

Fig. 5: Panels A, B and $\mathrm{C}$ depict the population breakdown by smoking category in Scn1, Scn2, and Scn3, respectively. Panels E, F, G, H, I and J illustrate fraction of CEU among CS, among FS and among NS in Scn2 and those fractions in Scn3, respectively. 
The effect of SN was modeled using multipliers $\left(m_{\text {net }}\right)$, and applied them to the baseline $r_{\mathrm{si}}$ and $r_{\mathrm{ECig}}$, respectively. The overall rate of SI and ECigUI of a particular agent were increased by $m_{\text {net }}$, relative to the rates in the baseline scenario. Without a specific mathematical model to quantify the effects of connected neighbors of a particular agent, the model employed a sigmoid function to describe the progression of the influence from the connected neighbors, which increases small at the beginning then accelerates fast and reaches the plateau. We, therefore, assumed $m_{\text {net }}$ follows a sigmoid function (Equation 1), where $f$ is the fraction of CS (or, correspondingly, CEUs) among its connected agents if $m_{\text {net }}$ is used to calculate the rate of SI or ECigUI, respectively, and $f_{0}, \alpha$ and $\gamma$ in Equation 11 are 0.25, 2.0 and 1.0, respectively. Similarly, in Equation 1 . $r_{\text {average }}$ represents the average rate of SI or rate of ECigUI of population for the calculation of rate of SI or ECigUI of this agent, respectively. The $r_{\text {average }} \mathrm{S}$ are re-calculated every year based on the smoking and ECig use status of the population at the beginning of each year.

$$
m_{\text {net }}=\frac{\alpha+e^{-\gamma \times\left(f-f_{0}\right)}}{\left(1+e^{-\gamma \times\left(f-f_{0}\right)}\right) \times r_{\text {average }}}
$$

Model Calibration $e_{\mathrm{si}}, e_{\mathrm{sc}}, e_{\mathrm{sr}}$ and $e_{\mathrm{ECig}}$ were calibrated to match the estimated PCS, prevalence of former smoker (PFS) and PCEU generated by the rates $\left(r_{\mathrm{si}}, r_{\mathrm{sc}}, r_{\mathrm{sr}}\right.$ and $\left.r_{\mathrm{ECig}}\right)$ in the baseline model, against historical data of 2013-2017 from CTADS [2]. The calibrated result of $e_{\mathrm{si}}, e_{\mathrm{sc}}, e_{\mathrm{sr}}$ and $e_{\mathrm{ECig}}$ is 1.088, 2.435, 1.51 and 7.898 , respectively.

Model Scenarios We examine here simulated population-level smoking behaviour change and ECig use under following three scenarios: smoking behavior in scenario one (Scn1) which is in absence of ECig use and the SN, smoking behavior in scenario two $(\mathrm{Scn} 2)$ which is under the use of ECig, and smoking behavior in scenario three (Scn3) which SN exists and supports the SI and ECigUI (Scn3). The outputs from these scenarios examined the difference in prevalence and incidence of smoking arising from considering ECigs as well as SN both separately and in combination. The simulation of Scn1 and Scn2 were run for 100 realizations, and simulation under Scn3 were run for 40 realizations with respect to the considerably large computation of SN in AnyLogic, with random seeds making each simulation run unique, then the means of the outputs of all runs were calculated for the comparison. Furthermore, to examine the statistical significance between the results from Scn1 and Scn2, we performed a Mann-Whitney-U test on the per-realization output (PCS), from the two scenarios.

Sensitivity Analysis To assess the sensitivity of model parameters on model outputs, we performed sensitivity analysis (SA) on the parameters such as the rate of ECigUC and the rate of ECigUR. The message transitions for ECigUC and ECigUR were replaced by the rate transitions. In the SA of the rate of ECiguC, the model assumed the rate of ECigUR is 1.0, and the range of the rate of ECigUC was 0.2 to 1.0 with a step of 0.2 for each iteration. Similarly, for the SA of the rate 
of ECigUR, the model assumed the rate of ECigUC is 1.0 and the rate of ECigUR had the same range and step with the rate of ECiguC in its SA experiment. The SA experiments examined the potential change of PCS resulting from changes in the value of the rate of ECigUC and the rate of ECigUR.

\section{Results}

Comparison between Scn1 and Scn2 Mean, median and standard deviation of the results for PCS, generated by the model realizations in Scn1, are 0.1438, 0.1440 and 0.0037 , respectively, and those from the model realizations in Scn2 are $0.1369,0.1374$ and 0.0074 , respectively. The results of a two-sided MannWhitney-U test for the results of two scenarios, $p<2.2 e^{-16}$, demonstrates that the distributions in the results of two scenarios differed significantly.

The message transitions in ECig use statechart were disabled in Scn1 and Scn2, therefore, in the stacked column chart showing the breakdown by smoking category (Figure 5 A, B and C), the agents were divided into four categories: CEU regardless of their smoking status, NS $\wedge$ NEU, CS $\wedge$ NEU, and FS $\wedge$ NEU, respectively. The portion of FS and NEU (23\%) in Scn1 is slightly higher than that $(21 \%)$ in Scn2, due to a large portion $(6 \%)$ of CEU, as shown in Figure 5 A and B. This reflects the fact that the FS in Scn1 is located within the FS $\triangle$ NEU category, whereas in Scn2 some of those individuals are located within the CEU category.

Comparison between Scn2 and Scn3 In Scn3, at the end of simulation, the maximum and minimum degree centrality of a given agent is 2 and 1 , respectively. With the presence of SN (in Scn3), as shown in Figure 5 C, the fraction of CEU in the population increased dramatically - rising from $6 \%$ in Scn2 to $61 \%$ in Scn3. With the exposure to ECig use from connected individuals or neighbors, people tend to initiate ECig use. The increased portion of CEU are mostly from the agents who were NSANEU. In Scn2, the fraction of CEU among CS and that among FS are similar, with value of $15 \%$ and $14 \%$ in Figure $5 \mathrm{E}$ and $5 \mathrm{~F}$, respectively, which are considerably larger than fraction of CEU among NS, as shown in Figure 5 G. In Scn3, the SN significantly increased the fraction of CEU among CS and FS, with the value of $86 \%$ and $65 \%$, respectively in Figure $5 \mathrm{H}$ and Figure 5, respectively, while the fraction of CEU among NS does not show obvious increase due to SN, compared with that of Scn2.

Sensitivity Analysis Results from the SA on the rate of ECigUC and the rate of ECigUR suggests that the PCEU and prevalence of former ECig user can substantially change the PCS, as shown in Figure 3 and 4 . Figure 3 demonstrates that when ECR is increased from 0.2 to 1.0 - holding invariant the value of ERR PCS are gradually increased, and PCEU decreases. The results in Figure 3 suggest that although incidence of SI is reduced by the lower PCEU, the decreased rate of SC and elevation in SR due to the decreased PCEU compensates for the decrease in the rate of SI. Similarly, the change in the rate of ECigUR also influences PCEU. Holding constant the rate of ECigUC, an increase in the rate of ECigUR generally 
increases the PCEU, but lowers the PCS, with a possible exception at the lowest levels of the rate of ECigUR. Results in Figure 4, the line from the rate of ECigUR of 0.2 having the lowest PCEU, reflect that agents were more likely to remain as CS.

\section{Discussion}

From the results in the three scenarios, the model demonstrates that ECig use and SN encourage agents to uptake ECig, therefore, shape population-level smoking behavior. Although the use of ECig increases the rate of SI, the combined effect of the increase in the rate of SC and the decrease in the rate of SR results a considerably large decline in PCS and increase in PFS. The results of SA further shows the PCS is sensitive to the ECig use behavior change. The outputs of the model largely depend on the feedback between smoking and ECig use, and interactions between agents. First, we assumed the rate of ECigUI of CS, FS and NS are in a declining order, specifically, the CS has highest rate of ECigUI compared with other smoking category. Second, if an individual is CS, being a CEU increases the probability of quitting smoking and staying in FS state, which means they have a relatively higher probability of using ECig as a SC tool. We assumed the ECig use helps greatly in SI for NS. Given the model results, fraction of CEU among NS is considerably lower, compared with CS and FS. Accordingly, as a combined result of the rate of SI, the rate of SC and rate of SR, the PCS is decreased due to ECig use. Furthermore, we assumed gender effect as divisors in the rate of ECigUI. Thus, the model behaves a relatively stronger influence from ECig use on smoking behavior. The effect of SN is modeled as a multiplier to the rate of ECigUI, which generates more CEU during simulation.

Despite fine resolution of the model, there are some limitations. First, the model is highly sensitive with the use of ECig, however, the model has no good assumption on the rate of ECigUC and the rate of ECigUR. Second, at this resolution, the model cannot capture the smoking episodes, dynamics of nicotine metabolism, allowing model to analyze whether ECig use helps in relieving nicotine cravings at fine-grained level as SC tool. Finally, the model assumes the effect of SN in a relatively simple way.

Although with some limitations, the model outcomes can provide some straightforward understanding of the complex feedback between smoking and ECig use at individual level, then allow us to analyze population-level smoking behaviour. Additionally, the model is also a useful tool for examining how SN influences smoking and ECig use, particularly among adolescents.

\section{References}

1. Population pyramids of the world from 1950 to 2100 (2017), https://www. populationpyramid.net/canada/2017/

2. Data about canadians' use of tobacco, alcohol and drugs. (2018-10-30), https://www. canada.ca/en/health-canada/services/canadian-tobacco-alcohol-drugs-survey.html 
3. Geography: Canada, province or territory (2019-01-26), https://www150.statcan.gc. $\mathrm{ca} / \mathrm{t} 1 / \mathrm{tbl1/en/tv}$.action?pid=1310071001

4. Fertility: Overview, 2012 to 2016 (June 5, 2018), https://www150.statcan.gc.ca/n1/ pub/91-209-x/2018001/article/54956-eng.htm

5. Axtell, R., Durlauf, S., Epstein, J.M., Hammond, R., Klemens, B., Parker, J., Song, Z., Valente, T., Young, H.P.: Social influences and smoking behaviour: Final report to the American Legacy Foundation (2006)

6. Center for Disease COntrol and Prevention: In: How Tobacco Smoke Causes Disease: The Biology and Behavioral Basis for Smoking-Attributable Disease: A Report of the Surgeon General

7. Chaturvedi, P., Mishra, A., Datta, S., Sinukumar, S., Joshi, P., Garg, A.: Harmful effects of nicotine. Indian Journal of Medical and Paediatric Oncology 36(1), 24 (2015)

8. Cherng, S.T., Tam, J., Christine, P.J., Meza, R.: Modeling the effects of e-cigarettes on smoking behavior. Epidemiology 27(6), 819-826 (Nov 2016)

9. Demick, B.: A high-tech approach to getting a nicotine fix (April 2009), http: //articles.latimes.com/2009/apr/25/world/fg-china-cigarettes25

10. Holford, T.R., Levy, D.T., McKay, L.A., Clarke, L., Racine, B., Meza, R., Land, S., Jeon, J., Feuer, E.J.: Patterns of birth cohort-specific smoking histories, 19652009. American Journal of Preventive Medicine 46(2), e31 - e37 (2014)

11. Leventhal, A.M., Strong, D.R., Kirkpatrick, M.G., Unger, J.B., Sussman, S., Riggs, N.R., Stone, M.D., Khoddam, R., Samet, J.M., Audrain-McGovern, J.: Association of electronic cigarette use with initiation of combustible tobacco product smoking in early adolescence. JAMA 314(7), 700-707 (08 2015)

12. McRobbie, H., Bullen, C., Hartmann-Boyce, J., Hajek, P.: Electronic cigarettes for smoking cessation and reduction. Cochrane Database of Systematic Reviews (Dec 2014)

13. Pisinger, C., Døssing, M.: A systematic review of health effects of electronic cigarettes. Preventive Medicine 69, 248 - 260 (2014)

14. Polosa, R., Caponnetto, P., Morjaria, J.B., Papale, G., Campagna, D., Russo, C.: Effect of an electronic nicotine delivery device (e-cigarette) on smoking reduction and cessation: a prospective 6-month pilot study. BMC Public Health 11(1) (Oct 2011)

15. Reid, J.L., Rynard, V.L., Czoli, C.D., Hammond, D.: Who is using e-cigarettes in Canada? nationally representative data on the prevalence of e-cigarette use among Canadians. Preventive Medicine 81, 180 - 183 (2015)

16. Rom, O., Pecorelli, A., Valacchi, G., Reznick, A.Z.: Are e-cigarettes a safe and good alternative to cigarette smoking? Annals of the New York Academy of Sciences 1340(1), 65-74 (Dec 2014)

17. U S Department of Health and Human Services:

18. Wills, T.A., Sargent, J.D., Gibbons, F.X., Pagano, I., Schweitzer, R.: E-cigarette use is differentially related to smoking onset among lower risk adolescents. Tobacco Control 26(5), 534-539 (Aug 2016)

19. Zhu, S.H., Zhuang, Y.L., Wong, S., Cummins, S., Tedeschi, G.: E-cigarette use and associated changes in population smoking cessation: evidence from US current population surveys. BMJ 358 (2017)

20. Zhuang, Y.L., Cummins, S.E., Y Sun, J., Zhu, S.H.: Long-term e-cigarette use and smoking cessation: a longitudinal study with us population. Tobacco Control 25(Suppl 1), i90-i95 (Oct 2016) 